post-infiltration. We also assessed heart rate and blood pressure on pre and post-procedure.

Results: Seventy-two participants were included with a mean age of 55.48 \pm 12.39 years (39-78), treated for an inflammatory rheumatic or degenerative disease $(21,51$ respectively), and receiving joint infiltration for the first time among 37. Sites of infiltrations were: wrist $=7$, elbow $=10$, shoulder $=17$, hip $=1$, knee $=22$, epidural $=6$, plantar heel $=9$. There were no significant differences in pre-procedural

VAS-Anx, VAS-pain, or physiological parameters between cases and controls. Cases had a significant decrease in VAS-Anx from pre to post-infiltration (Mean post-VAS-Anx=23.33/100, $p=0,017$ ) but not significant compared with controls $(p=0.297)$. Patients who have performed the breathing technique had no significant decrease in VAS-Pain from pre- to post-infiltration $(p=0.083)$ and compared with controls $(p=0.662)$. Physiological parameters showed a significant decrease in heart rate of cases from pre to post-infiltration $(p<0,0001)$ and compared with controls $(p=0,036)$, but no significant decrease in systolic or diastolic blood pressure from pre to post-infiltration and compared with controls. There were no correlations between all participants' VAS-Anx/VAS-Pain and age, gender, infiltration site or history and joint pain causes.

Conclusion: This study suggests that diaphragmatic breathing relaxation is an effective nonpharmacological intervention that could be used in controlling anxiety and experienced pain during joint infiltration.

Disclosure of Interests: None declared

DOI: 10.1136/annrheumdis-2021-eular.2861

\section{POS0156-HPR 'THE RIGHT ADVICE, IN THE RIGHT WAY AND AT THE RIGHT TIME': AN INNOVATIVE MULTIDISCIPLINARY APPROACH TO ADDRESS CLIENT-IDENTIFIED PARTICIPATION NEEDS IN EARLY INFLAMMATORY ARTHRITIS}

Y. Codd $^{1,2}$, R. Mullan ${ }^{2}$, D. Kane ${ }^{2}$, A. Coe ${ }^{2}$, T. Stapleton ${ }^{1} .{ }^{1}$ Trinity College Dublin, Discipline of Occupational Therapy, School of Medicine, Dublin, Ireland; ${ }^{2}$ Naas General \& Tallaght University Hospitals, Rheumatology, Dublin, Ireland

Background: Inflammatory arthritis (IA) is a complex life-long disease with negative consequences on occupational performance and significant impact on meaningful life roles. International guidelines for the clinical management of IA consistently recommend early intervention and a multidisciplinary (MDT) approach as the optimum method to address the medical, symptom and broader life impacts, however, barriers to timely access to the MDT is a common issue in clinical practice. Limited research has explored how clinical services support broader life impacts and participation restrictions associated with early disease as part of routine healthcare. Four rheumatology services in Ireland have recently reconfigured to provide an MDT-led early arthritis service which operates in parallel to traditional medical clinics. Staffed by nurses, occupational therapists, and physiotherapists this service provides assessment and management to clients with a new diagnosis of IA along a model of care within four to six weeks of diagnosis with mechanisms to follow the client over their first year of diagnosis. Objectives: To explore how a novel MDT-led early arthritis service addresses client-identified participation restrictions associated with early IA.

Methods: A qualitative description (QD) study was used to explore the perspectives of service providers and service users of this MDT-led early arthritis service. Data were gathered using one-off focus groups with service providers, and individual interviews with service users.

Results: All staff $(n=15)$ currently working in these services participated in the focus groups. Forty-three service users with IA participated in individual interviews (males $n=12$ : females $n=31$ ); diagnosis duration ranged from 5 to 24 months. QD principles and thematic analysis were used in data analysis.

Extensive limitations in everyday activity and restriction in participation in meaningful, age-appropriate life roles in early IA were outlined by service users and service providers. Participants described how the MDT-led model provided automatic and immediate access to services focussed on identification and management of occupation-based participation restrictions early in the disease. Participants described how the model used a flexible approach that ensured; ease of early access to a full MDT, prolonged support from the MDT, and a person-centred approach incorporating social prescribing and self-management principles. Service users emphasised the strength of the MDT approach of providing 'the right advice, in the right way and at the right time' as the most influential feature that assisted them to address participation restrictions and support positive health outcomes. This was accomplished by the service providers acting in a coordinated and interdisciplinary manner with a clear remit to address participation restrictions alongside the traditional symptom management approach. Findings highlight the feasibility of the MDT-led early arthritis model as an approach to address participation-based restrictions as part of routine healthcare that delivers on the rheumatology guidelines and was positively regarded by both service users and service providers.
Conclusion: Findings provide important insights into the role of an innovative early MDT intervention approach in addressing client-identified participation needs in early IA. This model maps onto the Irish rheumatology model of care (HSE, 2018), 'right place, right time' approach but importantly includes a 'right way' approach which is identified as influential in the effective delivery of client-focussed and client-centred care.

\section{REFERENCES:}

[1] HSE. (2018). Model of care for rheumatology in Ireland. Retrieved 15/09/2019, https://www.hse.ie/eng/about/who/cspd/ncps/rheumatology/achievements/ model-of-care-for-rheumatology-in-ireland.pdf

Acknowledgements: The authors, thank you to the research sites who facilitated this study \& the service providers and service users who partook in the research. Thank you to Kildare Branch of Arthritis Ireland who provided funding support for the research

Disclosure of Interests: None declared

DOI: 10.1136/annrheumdis-2021-eular.583

\section{POS0157-HPR CONSTRUCT VALIDITY OF THE OCTOPUS STRATIFICATION ALGORITHM FOR ALLOCATING PATIENTS WITH KNEE OSTEOARTHRITIS INTO CLINICALLY RELEVANT SUBGROUPS}

J. Knoop ${ }^{1}$, R. Ostelo ${ }^{1}$, M. Van der Esch ${ }^{2}$, A. De Zwart ${ }^{2}$, K. Bennell ${ }^{3}$, M. Van der Leeden ${ }^{2,4}$, J. Dekker ${ }^{4}{ }^{1}$ Vrije Universiteit Amsterdam, Health Sciences, Amsterdam, Netherlands; ${ }^{2}$ Reade, Centre for Rehabilitation and Rheumatology, Amsterdam, Netherlands; ${ }^{3}$ University of Melbourne, School of Health Sciences, Department of Physiotherapy, Melbourne, Australia; ${ }^{4}$ Amsterdam UMC (VUmc), Department of Rehabilitation, Amsterdam, Netherlands

Background: We recently developed a model of stratified exercise therapy [1], consisting of (i) a stratification algorithm allocating patients with knee osteoarthritis $(\mathrm{OA})$ into clinically relevant subgroups, and (ii) subgroup-specific exercise therapy. More specifically, in our model we distinguish the following subgroups with proposed underlying phenotypes: 'high muscle strength subgroup' representing a post-traumatic phenotype, 'low muscle strength subgroup' representing an age-induced phenotype and 'obesity subgroup' representing a metabolic phenotype. Objectives: In the present study, we aimed to validate the construct of this algorithm, focusing on 3 research questions: (i) are the proportions of patients in each subgroup similar across cohorts?; (ii) are the characteristics of each of the subgroups in line with their proposed underlying phenotypes?; (iii) are the effects of usual exercise therapy in the 3 subgroups in line with the proposed effect sizes? Methods: Data from five studies (four trials and one cross-sectional cohort) were used to validate the construct of our algorithm by 63 a priori formulated hypotheses regarding the research questions.

Results: Baseline data from a total of 1,211 patients with knee OA were analyzed for the first and second research question, and follow-up data from 584 patients who were part of an exercise arm within a trial for the third research question In total, the vast majority (73\%) of the hypotheses were confirmed. Regarding our first research question, we found similar proportions in each of the three subgroups across cohorts, especially for three cohorts. Regarding our second research question, subgroup characteristics were almost completely in line with the proposed underlying phenotypes. Regarding our third research question, unexpectedly, usual exercise therapy resulted in similar, moderate to large effect sizes for knee pain and physical function for all three subgroups.

Conclusion: This study suggests that our algorithm might be a valid instrument to allocate patients with knee OA into clinically relevant subgroups, as most of our a priori hypotheses could be confirmed. On the other hand, subgroups did not differ substantially in effects of usual exercise therapy, contrary to our expectation. An ongoing trial [1] will assess whether this algorithm accompanied by subgroup-specific exercise therapy improves clinical and economic outcomes. REFERENCES:

[1] Knoop J, Dekker J, van der Leeden M, de Rooij M, Peter WFH, van Bodegom-Vos L, van Dongen JM, Lopuhäa N, Bennell KL, Lems WF, van der Esch M, Vliet Vlieland TPM, Ostelo RWJG. Stratified exercise therapy compared with usual care by physical therapists in patients with knee osteoarthritis: A randomized controlled trial protocol (OCTOPuS study). Physiothe Res Int. 2020 Apr;25(2):e1819. doi: 10.1002/pri.1819. Epub 2019 Nov 28.

Disclosure of Interests: None declared

DOI: 10.1136/annrheumdis-2021-eular.1515

\section{POS0158-HPR UNDERSTANDING NURSE-LED CARE IN EARLY RA: INTERVIEW STUDY WITH RHEUMATOLOGY NURSE SPECIALISTS}

A. M. T. Sweeney ${ }^{1}$, J. Robson ${ }^{1}$, C. Flurey ${ }^{1}$, P. Richards ${ }^{1}$, C. Mccabe ${ }^{1}$, M. Ndosi ${ }^{1}$. ${ }^{1}$ University of the West of England, Faculty of Health and Applied Sciences, Bristol, United Kingdom 
Background: Nurse-led care in early RA is not well defined in the literature and the current recommendations.

Objectives: This study aimed to develop an understanding of what comprises nurse-led care in early RA from the perspective of rheumatology nurse specialists. Methods: This was a qualitative study using semi-structured telephone interviews with rheumatology nurse specialists in England (Summer 2020). Interviews were audio-recorded, transcribed verbatim and analysed using inductive thematic analysis.[1]

Results: Sixteen nurses were recruited and interviews lasted between 30 to 60 minutes. Seven themes were identified.

Early disease management

Care was characterised by evidence-based RA management provided by experienced nurse specialists with a high degree of autonomy, in the context of a rheumatology multidisciplinary team. The aims of care were to: start treatment, keep in treatment, educate and support.

'So treat to target...escalating treatment as necessary, and addressing any concerns that the patients might have' (CNS14)

Addressing psychosocial needs

Patients with early RA experience shock, fear, anger, grief and denial while feeling unwell with pain and fatigue. Nurses use a holistic, person-centred and empathetic approach to address psychosocial needs, building a working relationship, listening and creating trust.

'Because it all relates, and if they're stressed because they're not coping at work, then their arthritis isn't going to be so good. So everything relates to one another really' (CNS06)

\section{Monitoring treatment, disease impact and patient outcomes}

Nurses monitor disease activity and disease impact using validated outcome measures and by asking questions during the consultation. Good outcomes are disease control, managing disease impact, medication and side effects, wellbeing and keeping in work.

'When you get them stable, when you get them into remission, when they're happy, when they're feeling well, I think there's lots of ways you can measure that'... (CNS13)

\section{Coordinating care, referring and signposting}

Nurses coordinate care, refer to other health professionals and signpost patients to relevant services and charities. Lack of access to psychology expertise was highlighted.

'And whilst most of us have got some degree of understanding of...self management, or psychology... we're not psychologists' (CNS02)

Providing a 'lifeline'

Nurse-led telephone advice services provide a 'lifeline' for patients. If patients struggle, they can call and speak with a specialist who knows them and their RA well.

'The advice line has been a lifeline to them, to be able to speak to someone, to be able to get a response quickly to their questions, they feel very well supported, they know that they can always call us' (CNS16)

Service evaluation and auditing

The individual clinics are reviewed regularly. Patients are asked for feedback on their experience of appointments, if their needs were met and about changes to the service.

...I's really important to ask them initially what they expect to have from the consultation...We've always had really good feedback in general'... (CNS02) COVID-19 challenges and opportunities

The pandemic caused major disruptions to the services, prohibiting most faceto-face consultations which was an essential aspect of clinical assessments. Despite the challenges imposed by the pandemic, the services adapted fast, using telephone, video clinics and digital solutions, which streamlined procedures and improved documentation and communication.

'I do have to rely on them telling me what's going on, because I can't see it at the moment' (CNS14)

'With Covid we're doing it over the telephone, and we're getting them to watch the video [injection tutorial] before we have the appointment with them' (CNS04) Conclusion: Nurse-led care in early arthritis is a specialist service, addressing complex needs of patients, using evidence based and person-centred approaches. Innovation and service improvement are seen as part of the role. REFERENCES:

[1] Braun V, Clarke V. Successful Qualitative Research. First edition. London: SAGE 2013.

Disclosure of Interests: None declared

DOI: 10.1136/annrheumdis-2021-eular.555

\section{POS0159-HPR IMPACT OF SLEEP DISTURBANCES ON ELDERLY PATIENTS WITH RHEUMATOID ARTHRITIS}

M. Sellami ${ }^{1}$, O. Hamdi ${ }^{1}$, S. Miladi ${ }^{2}$, A. Fazaa ${ }^{2}$, L. Souabni ${ }^{2}$, K. Ouenniche ${ }^{2}$, S. Kassab ${ }^{2}$, S. Chekili ${ }^{2}$, K. Ben Abdelghani ${ }^{2}$, A. Laatar ${ }^{2}{ }^{1}$ Mongi Slim Hospital,
Rheumatology, Tunisia, Tunisia; ${ }^{1}$ Mongi Slim Hospital, Rheumatology, Tunisia, Tunisia

Background: Sleep disturbances have been reported in various rheumatic dis eases especially in the elderly. It may be caused by pain and depressive mood However, reports on the impact of sleep problems in rheumatoid arthritis (RA) activity and functional status were limited.

Objectives: To assess sleep quality in elderly patients with RA and its impact on disease activity and functional status.

Methods: This cross-sectional study included 70 RA patients aged $\geq 65$ years fulfilling the ACR/EULAR criteria. Sociodemographic data were collected. RA activity was assessed with the Disease Activity Score (DAS ${ }$ ) and functional status with the Health Assessment Questionnaire (HAQ). Sleep quality was assessed using Arabic translated versions of two indexes: the Insomnia Severity Index (ISI) and the Pittsburg Sleep Quality Index (PSQI). An ISI score of [8-14] [15-21], and [22-28] determined respectively mild, moderate, and severe insomnia. A PSQI score $>5$ determined poor sleep quality. ANOVA test was used to assess the relationship between $\mathrm{DAS}_{28}$ erythrocyte sedimentation rate (ESR), $\mathrm{HAQ}$, and sleep quality indexes.

Results: This study included 52 females and 18 males with a mean age of $68.3 \pm$ 25 years [65-81]. Seventy percent of patients were married, $27 \%$ were widowed and $2 \%$ were divorced. Seventy-one percent of patients were illiterate, $18 \%$ had primary education and $11 \%$ had secondary education. Eighteen percent of patients were employed whereas $34.7 \%$ were retired. A history of depression was noted in $16.5 \%$ of patients. The mean duration of RA was $17.4 \pm 5.2$ years. Eighty-five percent of patients were on conventional synthetic DMARD whereas $15 \%$ were treated with biologic treatment. The mean patient's global assessment of disease activity was $5.2 \pm 1$.3. The mean tender joint count and mean swollen joint count were $8 \pm 1.5$ and $5 \pm 1$ respectively. The mean DAS ${ }_{28}$ ESR was $4.7 \pm 0.9$. The mean HAQ was $2.4 \pm$ 0.45 . Poor sleep quality was detected in $84 \%$ of cases according to the PSQI score. Mild insomnia was detected in $46 \%$ of cases, moderate insomnia in $34 \%$ of cases, and severe insomnia in $12 \%$ of cases. RA activity was higher in patients with poor sleep quality: the mean DAS ${ }_{28}$ ESR was 5.2 in patients with severe insomnia, 4.82 in moderate insomnia, and 4.13 in mild insomnia; $p=0.00$ respectively. The mean ESR was $31.5 \mathrm{~mm}$ in patients with severe insomnia, $22.1 \mathrm{~mm}$ in moderate insomnia and $10.6 \mathrm{~mm}$ in mild insomnia; $\mathrm{p}=0.01$ respectively. Furthermore, the higher the PSQI was, the higher DAS ${ }_{28} E S R$ is $(p=0.01)$. However, no association was found between poor sleep quality and joint count, swollen joint count, CRP, and HAQ.

Conclusion: Disease activity was a major contributor to poor sleep quality in elderly patients with RA. Functional status however wasn't associated with insomnia. Physicians should include sleep in the clinical assessment of RA patients to improve their quality of life.

Disclosure of Interests: None declared

DOI: 10.1136/annrheumdis-2021-eular.408

\section{POS0160-HPR THE CARE PROCESS OF HIP AND KNEE OSTEOARTHRITIS: GIVING AN ACCOUNT OF PATIENTS' EXPERIENCE}

S. Battista ${ }^{1,2}$, M. Manoni ${ }^{1}$, A. Dell'isola ${ }^{2}$, M. Englund ${ }^{2}$, A. Palese ${ }^{3}$, M. Testa ${ }^{1}$.

${ }^{1}$ University of Genova, Department of Neurosciences, Rehabilitation, Ophthalmology, Genetics, Maternal and Child Health, Savona, Italy; ${ }^{2}$ Lund University, Clinical Epidemiology Unit, Orthopedics, Department of Clinical Sciences, Lund, Sweden; ${ }^{3}$ University of Udine, School of Nursing, Department of Medical Sciences, Udine, Italy

Background: The care process is often a complex and intimate process experienced by patients. Osteoarthritis $(\mathrm{OA})$ care is usually characterised by multimodal interventions that consider the broader array of symptoms and functiona limitations and often require a high level of patients' compliance. Despite efforts to improve the quality of care of patients suffering from $O A$, and the publication of state-of-the-art clinical practice guidelines [1], the quality of the care process, as experienced by patients, seems to be suboptimal [2]. Hence, it is essential to investigate how patients experience this process to highlight potential elements that can enhance or spoil it to optimise the care quality.

Objectives: To explore the patients' experience of the received OA care process. Methods: Qualitative study, 10 semi-structured interviews were performed. The interview guide was created by a pool of healthcare professionals (physiotherapists, psychologists, nurses) and expert patients. It investigated the emotional experience, beliefs, expectations, perceived barriers and facilitators towards conservative treatments perceived by patients suffering from $O A$. The interviews lasted approximately one hour, were transcribed verbatim and analysed independently by two authors, who labelled their core parts to find categories and subcategories. A theme-based analysis was performed following an ecologica paradigm, naturalistic epistemology, philosophy of phenomenological research. Results: Our analysis revealed 7 main categories with several subcategories (Fig. 1). 1) Uncertainty as some patients perceived treatment choice not to be 